Doris Franco-González; Darwin Gabriel García-Herrera; Claudio Fernando Guevara-Vizcaíno; Juan Carlos Erazo-Álvarez

http://dx.doi.org/10.35381/r.k.v5i5.1050

\title{
Scratch para la enseñanza de Lenguaje de Programación en Primero de Bachillerato
}

Scratch for teaching programming language in the first year of high school

\author{
Doris Franco-González \\ doris.franco.98@est.ucacue.edu.ec \\ Universidad Católica de Cuenca, Azogues \\ Ecuador \\ https://orcid.org/0000-0002-4027-3885 \\ Darwin Gabriel García-Herrera \\ dggarciah@ucacue.edu.ec \\ Universidad Católica de Cuenca, Azogues \\ Ecuador \\ https://orcid.org/0000-0001-6813-8100 \\ Claudio Fernando Guevara-Vizcaíno \\ cguevarav@ucacue.edu.ec \\ Universidad Católica de Cuenca, Cuenca \\ Ecuador \\ https://orcid.org/0000-0003-3593-0606 \\ Juan Carlos Erazo-Álvarez \\ jcerazo@ucacue.edu.ec \\ Universidad Católica de Cuenca, Cuenca \\ Ecuador \\ https://orcid.org/0000-0001-6480-2270
}

Recibido: 15 de septiembre de 2020

Revisado: 19 de octubre de 2020

Aprobado: 15 de noviembre de 2020

Publicado: 01 de diciembre de 2020 
Revista Arbitrada Interdisciplinaria KOINONIA

Año 2020. Vol V. N5. Especial II: Educación

Hecho el depósito de Ley: FA2016000010

ISSN: 2542-3088

FUNDACIÓN KOINONIA (F.K). Santa Ana de Coro. Venezuela.

Doris Franco-González; Darwin Gabriel García-Herrera; Claudio Fernando Guevara-Vizcaíno; Juan Carlos Erazo-Álvarez

\title{
RESUMEN
}

El objetivo de este trabajo es analizar el Scratch para la enseñanza de Lenguaje de Programación en Primero de Bachillerato del Ecuador. La metodología se generó desde un enfoque mixto de alcance descriptivo transversal. El desarrollo de esta habilidad se ha tornado difícil en el triángulo educativo por su complejidad, debido a varios factores, uno de ellos son los gobiernos de turno por su pésima asesoramientos en el sistema educativo, no se pega a la realidad social perjudicando a niños y adolescentes principalmente de bajos recursos económicos, otro de las causas es la escasa utilización de herramientas tecnológicas por parte de los docentes, una sociedad digitalizada genera proyectos transformadores de políticas, económicas y sociales.

Descriptores: Enseñanza multimedia; tecnología educacional; aprendizaje en línea. (Palabras tomadas del Tesauro UNESCO).

\begin{abstract}
One of the problems in the educational field is that children and adolescents are not creative, they do not like to analyze, they are not interested in reading. Traditional teaching is one of the factors that cause this problem. Working with educational tools, in the matter of programming, would help the development of algorithmic logic and in turn the resolution of problems, tasks that are carried out through systematic analysis. that no positive results are evidenced due to poor academic performance. For this, it is necessary for the teacher to innovate and use technological tools, and thereby prevent students from finding themselves in a passive way where they only respond to what is planned. The poor development of algorithmic cognitive skills without the complexities of syntax, such as traditional programming languages, represents a failure in their university and work life.
\end{abstract}

Descriptors: Multimedia instruction; educational technology; electronic learning electronic learning. (Words taken from the UNESCO Thesaurus). 


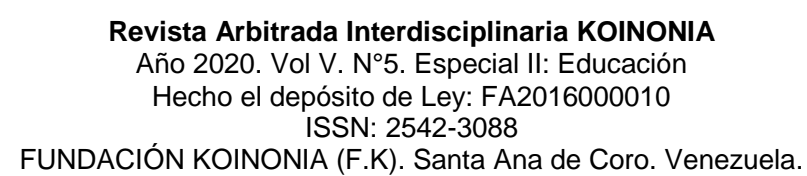

Doris Franco-González; Darwin Gabriel García-Herrera; Claudio Fernando Guevara-Vizcaíno; Juan Carlos Erazo-Álvarez

\section{INTRODUCCIÓN}

Scratch como un escenario de aprendizaje que responde a las necesidades de formación actuales de los estudiantes, unos de los factores que hay en aula es la falta de motivación la falta de interés de ambos escenarios docente y estudiantes, vemos un proceso formativo en el contexto ecuatoriano, que no da respuesta a las necesidades y requerimientos de los estudiantes; de acuerdo a las estadísticas del (Instituto Nacional de Evaluación Educativa (INEVAL) 2019), menciona que hay un avance significativo en estos últimos años en las materias de Lengua, Matemáticas y Ciencias Naturales, pero no llegan a las expectativas de nuestros estudiantes que es el ingreso a las universidades, se reflejan los resultados en la prueba quiero ser bachiller, sueños de una juventud frustrado.

El rol de docentes, desde la básica se debe centrar en el desarrollo del pensamiento lógico computacional, tarea de todas las disciplinas académicas, el fortalecimiento de la competencia digital el uso de nuevas herramientas y el recurso tecnológico, en niños y a jóvenes da como resultados a estudiantes críticos reflexivos, capaces de resolver problemas en su diario vivir.

Un docente creativo presto al cambio tendrá alumno motivados, dinámicos, entusiastas creativos la herramienta Scratch encierra estas habilidades, alternativa para conseguir la atención, el entusiasmo a la programación, siendo creadores de sus propios paquetes de software y no solo consumidores del mismo, aplicando una metodología basado en proyectos, y trabajo colaborativo y recreativo.

En Ecuador en el 2016 se estableció Infocentros Comunitarios iniciativa que se realiza en forma mundial dirigida a niñas y a niños con el único propósito del fortalecimiento lógico, lamentablemente este proyecto no tiene secuencia por parte de la cartera de Educación.

El objetivo de este trabajo es analizar el Scratch para la enseñanza de Lenguaje de Programación en Primero de Bachillerato del Ecuador. 


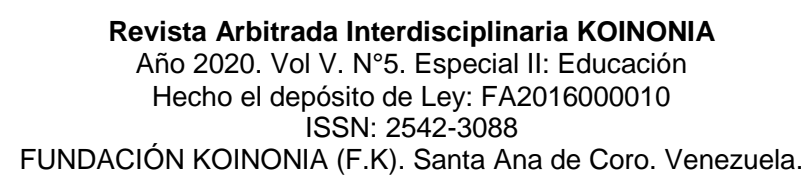

Doris Franco-González; Darwin Gabriel García-Herrera; Claudio Fernando Guevara-Vizcaíno; Juan Carlos Erazo-Álvarez

\section{Referencial teórico}

En la revisión de la literatura se establece que las estadísticas de varios países demuestran que el uso de herramientas computacionales y Scratch es importante, uno de los autores es (Miranda-Pinto, 2019), quien menciona que el uso de la herramienta en niños, mejora la adquisición de conceptos computacionales básicos y de su aplicación, indispensable para el aprendizaje de la programación, considerada de alta complejidad. En la era digital que vivimos, es indispensable una formación tecnológica, los estudiantes deben estar listos para un ser productivos y eficientes en el campo laboral, de ahí surge la necesidad de desarrollar su lógica desde muy temprana edad. Esto lo ratifican (Pindo, et al., 2016), quienes realizaron una propuesta en Argentina para los estudiantes de primaria, recalcaron las ventajas de que la enseñanza de las ciencias computacionales se enfocara en que los estudiantes crearan sus propias herramientas, utilizando diferentes juegos de robótica. Recomiendan el Aprendizaje Basado en Proyecto (ABP), utilizando como motivación la creación de juegos.

(Cózar-Gutiérrez, et al., 2019), analizaron en todos los países europeos: el uso de la programación y codificación informática, desde el punto de vista de los docentes. Encontraron que en muchos de los países se realizan competencias para atraer al estudiantado por medio de la robótica, a pesar de que en algunos no es obligatorio dentro del currículo y en algunos casos los maestros deben capacitarse por sí mismos. Los investigadores concluyen que generalmente la programación y la robótica atraen a los estudiantes más calificados y resultan atractivos y gratificantes para ellos.

En América el Sur, (Monjelat \& San Martín, 2016), han analizaron el uso de Scratch en experiencias educativas argentinas, concluyeron que su interfaz era adecuado para la exigencia del aprendizaje de computación y que debía adecuarse a todos los contextos poblacionales, considerando las opiniones de los participantes. Por otro lado, (Vidal, et al., 2015), comentan que en Chile realizaron experimentos para detectar el avance académico con estudiantes de nivel secundario, en el uso de la programación de Scratch, se llegó a la conclusión de que desarrolla la lógica algorítmica. 


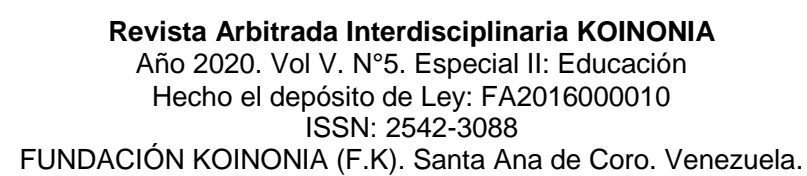

Doris Franco-González; Darwin Gabriel García-Herrera; Claudio Fernando Guevara-Vizcaíno; Juan Carlos Erazo-Álvarez

Cuando se habla de tecnología en Ecuador, se registra un avance en términos de adquisición de tecnología. A partir de 2006 el Estado integra a las Tecnologías de la Información y comunicación [TIC], en el ámbito educativo. (Peñaherrera-León, 2012), la autora resalta el Plan De tal palo, tal astilla, en el que el Ministerio de Educación dotó de computadoras y el software Scratch y la robótica, a varias escuelas públicas en la Provincia del Oro, lamentablemente los docentes recibieron formación en TIC, pero no en programación.

Por otro lado, (García-Jiménez \& Roig-Vila, 2018), analizan las ventajas de utilizar entornos de programación para el aprendizaje de la computación en un nivel de educación superior. Recomiendan el uso de Scratch como un paso previo al aprendizaje computacional y la necesidad de incentivar a los estudiantes a utilizarlo para diferentes propósitos, primero como prácticas simples para poco a poco desarrollar proyectos más ambiciosos, en cualquier ámbito. Concluyen que con Scratch, el alcance del pensamiento computacional fue mayor.

Por otro lado, (Marín-Gutiérrez, et al., 2014), analizaron el dominio de la tecnología tanto de docentes como de estudiantes en las ciudades de Loja y Morona, indican que las tecnologías son un factor clave para individualizar la enseñanza, ya que los estudiantes pueden adaptar su proceso y su ritmo de aprendizaje, unos de los aciertos que se dio por parte del Ministerio de Educación es la inserción de las tecnologías a los colegios tanto a los jóvenes y a sus profesores, mediante el uso de las TIC, se lo calificó como sociedad del conocimiento.

Una de las problemáticas que se tiene en el aula, son estudiantes apáticos y desinteresados en su proceso educativo y como resultado, con un desarrollo bajo en su lógica algorítmica (Aguilar-Yalamá \& Pinto-Garzón, 2015), menciona que el desarrollo de competencias digitales es indispensable para solucionar aspectos de la vida académica, profesional y laboral de los estudiantes. La tecnología no es suficiente por sí misma, es necesario que estudiante y docente se formen en el desarrollo de un pensamiento digital. 


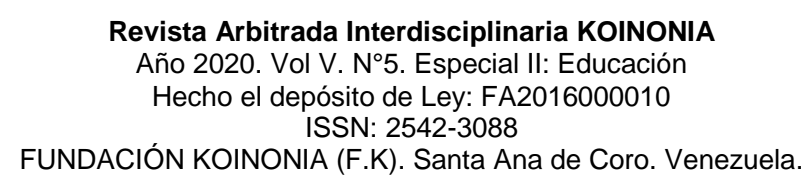

Doris Franco-González; Darwin Gabriel García-Herrera; Claudio Fernando Guevara-Vizcaíno; Juan Carlos Erazo-Álvarez

El cambio de las tecnologías en la actualidad es muy rápido y cada vez más globalizante. Según menciona (Ziehlke \& Talay, 2017), Scratch es un lenguaje de programación que apareció por primera vez en 2003, para niños desde los 8 años y ya para 2013, tenía su versión online. Para estos autores es un lenguaje de interfaz simple que resulta atractivo y adictivo para el estudiante, permitiéndole además trabajar de forma colaborativa con cualquier persona del mundo.

A decir de (Brennan \& Resnick, 2012), siendo este último director del proyecto de Scratch, este programa desarrolla el pensamiento computacional. Consiste en armar bloques de estructuras, en ordenar pedazos de códigos, que a modo de un rompecabezas buscan generar diferentes escenarios significativos para el aprendiz. Por sus características es útil también para cualquier docente que quiera crear juegos para Gamificar su clase. A nivel del país, el uso de las tecnologías lleva un retraso con respecto a los países de primer mundo. El uso de Scratch como un lenguaje de programación va adquiriendo importancia desde hace 5 años.

El aprendizaje de lenguajes de Programación, en un sistema instruccional, no ha permitido que se desarrollen las capacidades de programación, elemento indispensable que sirve para saber cómo y cuándo emplear o resolver un determinado problema, Ante esta realidad, es necesario cambiar los sistemas de enseñanza para que los estudiantes puedan por sí mismos diseñar sus entornos digitales y aprender.

Desde el aporte de (Balanskat \& Engelhardt, 2015), una de las visiones que adoptaron muchos países, es que la materia de programación sea de carácter transversal, de ahí viene la necesidad de una disciplina llamativa para los estudiantes, surgiendo el Scratch por su representativo enfoque sencillo, diverso y divertido hacia la programación. La necesidad de tener a niños y adolescentes creativos que aprendan a programar sin procesos arduos y cansados hizo que naciera Scratch. Desde el contexto educativo ecuatoriano, no podemos dejar de analizar la transformación de nuestro vivir, es requisito de un estudiante de programación tener un pensamiento lógico desarrollado. 


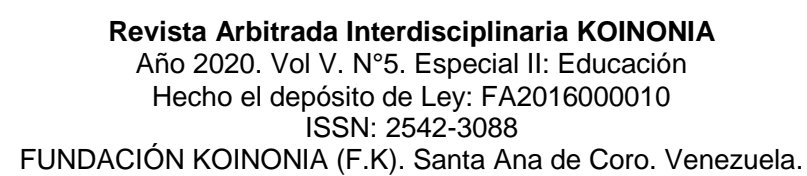

Doris Franco-González; Darwin Gabriel García-Herrera; Claudio Fernando Guevara-Vizcaíno; Juan Carlos Erazo-Álvarez

Otro de los factores importantes es la conectividad y más aún cuando la actualidad lo exige, los docentes deben dar las directrices para que los estudiantes sean investigativos y de conocimiento productivo, he aquí el término "aprender a aprender". Una de las limitaciones para el uso de Scratch para programación, son los limitados conocimientos digitales por parte de los docentes.

La Gamificación, según (Contrera-Espinoza, 2017), se puede aprovechar el sistema de recompensas, de retos, que usualmente la Gamificación tiene al ejecutar cada evento alcanzando el interés de la competencia, Scratch se constituye entonces, en una estrategia que puede utilizarse no sólo en Gamificación, sino en otras metodologías activas para conseguir la atención y motivación de los estudiantes.

De (Monjelat \& San Martin, 2016), mencionan que el aprendizaje de la programación computacional permite desarrollar diferentes habilidades, entre ellas: el pensamiento abstracto, el lógico matemático, la capacidad de tomar decisiones propias, ser creativos y aprender a trabajar en equipo. Estas competencias le servirán para un bueno desempeño en todas las asignaturas, no sólo en Programación, y más importante, para su desenvolvimiento laboral.

Los investigadores de Scratch están trabajando para facilitar el uso del lenguaje para la educación y hacerlo cada vez más accesible e intuitivo. De esta forma tanto docentes como estudiantes, ya sea en computadora u online, podrán compartir sus creaciones de forma fácil y divertida.

Aplicando una metodología que nos ayude a obtener un alcance significativo e innovador se utiliza el Aprendizaje Basado en Proyectos. Como manifiestan (Quiles-Carrillo, et al., 2019) esta metodología fomenta "el aprendizaje autónomo, la capacidad de autoevaluación, la planificación del tiempo, el trabajo por proyectos o la capacidad de expresión oral y escrita." (p.2) y consiste en la motivación y participación académica para construir saberes. Para alcanzar el objetivo se requiere de un trabajo en equipo, colaborativo. 


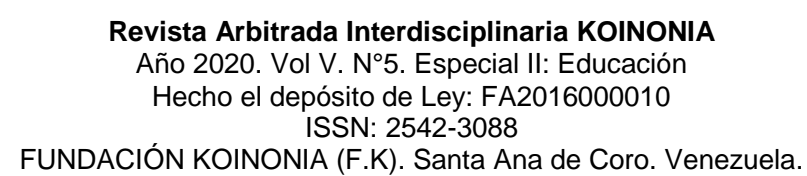

Doris Franco-González; Darwin Gabriel García-Herrera; Claudio Fernando Guevara-Vizcaíno; Juan Carlos Erazo-Álvarez

Se evalúa el proceso de programación, basándose en que el estudiante adquiera la competencia de aprender a aprender. Para aplicar este método se requiere de un aporte práctico, de forma cualitativa y cuantitativa desde un punto constructivista. Previa a la obtención de notas en forma grupal e individual, debe darse a conocer a los estudiantes la rúbrica de evaluación, aplicar la coevaluación, identificar errores, obtener resultados cuantitativos y cualitativos y culminar con pequeños proyectos.

Como lo señalan los autores, la herramienta Scratch permite el desarrollo del pensamiento computacional, de allí su importancia. Para ello es necesario que el docente innove y utilice herramientas tecnológicas, y con ello evitar que los estudiantes se encuentren de una forma pasiva donde respondan sólo a lo planificado. El poco desarrollo de habilidades cognitivas algorítmicas sin las complejidades de sintaxis, como son los lenguajes de programación tradicionales, representa un fracaso en su vida universitaria y laboral.

Desarrollar en los estudiantes competencias digitales, es lo mejor que se puede hacer por una sociedad. A decir (Terneus-Páezet al., 2019), en España, "un 68\% de las empresas detecta una brecha de conocimiento entre tecnología y digitalización". Según las autoras, es necesario que los graduados de las universidades posean competencias digitales, la capacidad de actualizarse todo el tiempo y de adaptarse a los cambios permanentes en los entornos de trabajo, recomiendan una conexión permanente entre centros educativos y empresas.

No tenemos este tipo de análisis en nuestro país, pero es evidente que el razonamiento es importante en la solución de problemas. Scratch es una solución constructivista para el aprendizaje, ya que genera conflicto y la búsqueda inmediata en la forma de resolver el problema, haciendo del estudiante una persona activa y autónoma. Las autoras resaltan el estudio de casos de personas con necesidades educativas especiales motoras e intelectuales, que han logrado aprender más y mejor con este lenguaje de programación. 


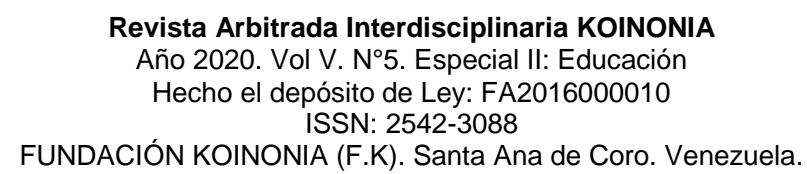

Doris Franco-González; Darwin Gabriel García-Herrera; Claudio Fernando Guevara-Vizcaíno; Juan Carlos Erazo-Álvarez

Con el lenguaje Scratch podrán desarrollarse muchas destrezas y habilidades, se aprende jugando, utilizando animaciones y simulaciones para su fácil comprensión, los docentes deben ver las bondades de aprender y utilizar este lenguaje de programación, ya que coadyuvará a que los estudiantes sean críticos, reflexivos, creativos y comunicativos, y además permitirá que las clases docentes sean divertidas y el aprendizaje sea individualizado. El trabajar con Scratch las ventajas que brinda para mejorar el aprendizaje de los estudiantes en la materia de lenguajes de programación a base de pequeños proyectos, ayudando al desarrollo lógico computacional a base algorítmicas.

\section{METODOLOGÍA}

La metodología se generó desde un enfoque mixto de alcance descriptivo transversal. Para el apartado cuantitativo se empleó un diseño cuasi experimental de pre y pos test, en una población de 58 estudiantes del primer año de bachillerato con 4 paralelos A-B en pre-test y C-D en post-test del Colegio Henríquez Coello de la Ciudad de Machala, siendo el tratamiento aplicado la herramienta tecnológica Scratch. Se empleó como técnica de recolección de información, la encuesta y un instrumento tipo prueba de 13 ítems con la valoración de excelente, muy bueno, bueno, regular, malo; siendo validado por cálculo de coeficiente Alfa de Cronbach 0,799. Para el segmento cualitativo, se trabajó con entrevista a grupo focal, en este caso, la población fueron 4 docentes especializados en tecnología educativa de la provincia de El Oro - Ecuador, aplicándose el análisis de contenido para formular categorías. 
Revista Arbitrada Interdisciplinaria KOINONIA

Año 2020. Vol V. N5. Especial II: Educación

Hecho el depósito de Ley: FA2016000010

ISSN: 2542-3088

FUNDACIÓN KOINONIA (F.K). Santa Ana de Coro. Venezuela.

Doris Franco-González; Darwin Gabriel García-Herrera; Claudio Fernando Guevara-Vizcaíno; Juan Carlos Erazo-Álvarez

\section{RESULTADOS}

Se detallan los principales resultados obtenidos:

\section{Tabla 1.}

Relación entre Se concentran en las actividades que realizan y Te gusta la materia de Programación.

\begin{tabular}{cccccccc}
\hline & & \multicolumn{3}{c}{ Te gusta la materia de Programación } & \multirow{2}{*}{ Total } \\
& & Mucho & Poco & Muy Poco & Nada & \\
\hline \multirow{3}{*}{ Se concentran en las } & Excelente & 23 & 1 & 0 & 0 & 24 \\
actividades que realizan & Muy Bueno & 3 & 2 & 0 & 0 & 5 \\
& Bueno & 15 & 3 & 1 & 1 & 20 \\
& Regular & 9 & 0 & 0 & 0 & 9 \\
\hline Total & & 50 & 6 & 1 & 1 & 58 \\
\hline
\end{tabular}

Pruebas de chi-cuadrado

\begin{tabular}{lccc}
\hline & Valor & Gl & Sig. asintótica (bilateral) \\
Chi-cuadrado de Pearson & $11,432^{\mathrm{a}}$ & 9 & 0,247 \\
Razón de verosimilitudes & 11,268 & 9 & 0,258 \\
Asociación lineal por lineal & 0,830 & 1 & 0,362 \\
\hline N de casos válidos & 58 & &
\end{tabular}

a. 13 casillas $(81,3 \%)$ tienen una frecuencia esperada inferior a 5 . La frecuencia mínima esperada es ,09.

\section{Fuente: Elaboración propia}

La tabla1 muestra el análisis de Chi cuadrado el valora 0,247 mayor a 0,05 la misma que se asume una hipótesis asociada entre la frecuencia que se concentran en que realizan y el gusta de la materia de programación, se observa un considerable número de estudiante que se encuentra en un nivel medio, utilizando la herramienta se espera resultados de medio superior. 
Revista Arbitrada Interdisciplinaria KOINONIA

Año 2020. Vol V. N5. Especial II: Educación

Hecho el depósito de Ley: FA2016000010

ISSN: 2542-3088

FUNDACIÓN KOINONIA (F.K). Santa Ana de Coro. Venezuela.

Doris Franco-González; Darwin Gabriel García-Herrera; Claudio Fernando Guevara-Vizcaíno; Juan Carlos Erazo-Álvarez

Tabla2.

Resultado global de pre y post test.

\begin{tabular}{ccccccccc}
\hline & Ayu.demás & \multicolumn{2}{c}{ Asist. } & \multicolumn{2}{c}{ Act.traba } & \multicolumn{2}{c}{ Motivados } \\
\hline & Test & & Test & & Test. & \multicolumn{2}{c}{ Test } & \\
\hline $\mathrm{N}$ & Pre. & Post. & Pre. & Post. & Pre. & Post & Pre. & pos \\
\hline $\begin{array}{c}\text { Media } \\
\text { Desviación }\end{array}$ & 2,26 & 27 & 31 & 27 & 31 & 27 & 31 & 27 \\
$\quad$ típ & 1,505 & 1,203 & 1,274 & 1,010 & 1,175 & 1,013 & 1,267 & 1,182 \\
$\begin{array}{c}\text { Error típ. de } \\
\text { la media }\end{array}$ & 0,270 & 0,232 & 0,229 & 0,194 & 0,211 & 0,195 & 0,228 & 0,227 \\
\hline
\end{tabular}

Fuente: Elaboración propia.

En relación a la variable ayudas y colaboras con los demás se analizar en el pretest la media 2,26 y el poste test de 2,70 se observa que los estudiante si demuestra la colaboración con sus compañeros, obtenido en t studies $(0,000)$ un normal esperado por lo tanto la hipótesis es afirmativa, en la variable asiste con regularidad se observa en el pretest 210 y el postest 241 , en la prueba t nos da un índice $(0,000)$ la asistencia de los estudiante a clase es importante se encuentra con una hipótesis moderada después de trabajar con la herramienta Scratch llama la atención y la curiosidad de los estudiantes, en la variable Activo y trabajador en el pretest 1,77 postest 2,56 nos da índice de normal $(0,000)$ demuestra un avance significativo el indicador Motivación pretest 2,16 postest 2,63 en la prueba t con un índice $(0,000)$ nos da un avance progresivo.

Con el análisis de los resultados la utilización de la herramienta Scratch para el desarrollo de la lógica computacional y el aprendizaje del lenguaje de programación se observa un porcentaje considerable de estudiantes que no les agradaba la materia por su complejidad, en el postest de esta variable nos revela una significativa diferencia, aplicando ABP ((Aprendizaje Basado en Proyectos) y utilización de la herramienta 
Revista Arbitrada Interdisciplinaria KOINONIA

Año 2020. Vol V. N5. Especial II: Educación

Hecho el depósito de Ley: FA2016000010

ISSN: 2542-3088

FUNDACIÓN KOINONIA (F.K). Santa Ana de Coro. Venezuela.

Doris Franco-González; Darwin Gabriel García-Herrera; Claudio Fernando Guevara-Vizcaíno; Juan Carlos Erazo-Álvarez

Scratch se obtiene a estudiantes motivados y con resultados satisfactorios en su razonamiento lógico.

Las variables y sus dimensiones en la investigación para la obtención de información nos dan que la herramienta Scratch programación orientado a objetos, es diferente a otros por su interfaz interactiva y sus múltiples escenarios, la presentación de instrucción se da de forma de bloque o legos que encaja unos sobre otros, las características más relevantes son: sentencias de entrada y salida, movimientos, variable, operaciones, tomas de decisiones, sentencias repetitivas, detector de errores entre otros unas de las piezas claves de esta investigación es el docente, se realizó una entrevista escrita para conocer de qué forma trabajan con la materia de programación, docentes del área informática de la ciudad de Machala, dando diversas opiniones y las más relevante son:

\section{Tabla 3.}

Análisis cualitativo.

\begin{tabular}{|c|c|}
\hline Categoría & Segmento \\
\hline $\begin{array}{l}\text { ¿Por qué no les agrada la materia de } \\
\text { programación? }\end{array}$ & $\begin{array}{l}\text { "Porque ya no es muy llamativa la asignatura" } \\
\text { "Porque es un lenguaje desconocido y complejo de } \\
\text { aprender" } \\
\text { "seria porque no les gusta razonar } \\
\text { Es una asignatura/módulo en la que el estudiante } \\
\text { necesita saber pensar, pero con unas buenas } \\
\text { técnicas de enseñanza, participación activa se } \\
\text { podrían encariñar con la materia. } \\
\text { porque es una materia de razonamiento lógico y } \\
\text { lamentablemente no están enseñados a eso } \\
\text { No les gusta desarrollar la lógica. } \\
\text { seria porque no les gusta razonar }\end{array}$ \\
\hline ¿Motivación en la clase de programación? & $\begin{array}{l}\text { Participación activa, grupal, lluvia de ideas iniciar con } \\
\text { juegos de razonamiento, indicarles lo que pueden } \\
\text { Ilegar a crear mediante los lenguajes de } \\
\text { programación } \\
\text { Clases participativas con desarrollo de proyectos } \\
\text { aplicables a su realidad } \\
\text { Los motivos con un punto para que resuelvan el } \\
\text { ejercicio } \\
\text { Realizar una dinámica relacionada al tema y hacer la } \\
\text { clase más práctica que teórica }\end{array}$ \\
\hline
\end{tabular}


Revista Arbitrada Interdisciplinaria KOINONIA

Año 2020. Vol V. N5. Especial II: Educación

Hecho el depósito de Ley: FA2016000010

ISSN: 2542-3088

FUNDACIÓN KOINONIA (F.K). Santa Ana de Coro. Venezuela.

Doris Franco-González; Darwin Gabriel García-Herrera; Claudio Fernando Guevara-Vizcaíno; Juan Carlos Erazo-Álvarez

\begin{tabular}{ll}
\hline & $\begin{array}{l}\text { Mostrarle sistema informático de alta gama y que se } \\
\text { utiliza a nivel mundial }\end{array}$ \\
\hline ¿Conoces la herramienta Scratch, el haz & $\begin{array}{l}\text { Desconozco } \\
\text { utilizado? }\end{array}$ \\
& $\begin{array}{l}\text { Si, Apropiadamente para el desarrollo de habilidades } \\
\text { mentales en el desarrollo de la programación, para } \\
\end{array}$ \\
& un buen inicio \\
\hline
\end{tabular}

Fuente: Elaboración propia.

Se puede observar resultados diversos en la encuesta, en cuanto al porqué no les agrada la materia de Programación, la respuesta más significativa es que no les agrada razonar, en este sentido el razonamiento lógico, que hace uso del entendimiento para pasar de unas proposiciones a otras, partiendo de lo ya conocido o de lo que se cree conocer a lo desconocido o menos conocido.

Los estudiantes que no desarrolle su lógica genera una problemática que tendrán consecuencias permanentes en el ámbito escolar y profesional, es tarea de todas las áreas el hacerlo. Los docentes hacen su esfuerzo para llamar la atención con varias dinámicas sin tener resultados favorables, hay un porcentaje de docente que no conoce la herramienta Scratch, por ende, ejercen clases tradicionales.

\section{PROPUESTA}

La utilización de una herramienta desde la básica superior ayudaría a mitigar los problemas en el primer año de bachillerato, se trabajaría con estudiante desarrollada su lógica computacional y la aceptación a la materia de Programación. El proceso de enseñanza-aprendizaje de la programación a través de Scratch es entonces un reto doble, que permitirá que el estudiante sea más productivo y vea su educación como una oportunidad de competir a nivel tecnológico, se propone 3 aspectos que son:

1.- Currículo - Docente

2.- Recursos pedagógico

3.- Alfabetizar digitalmente al estudiante, 


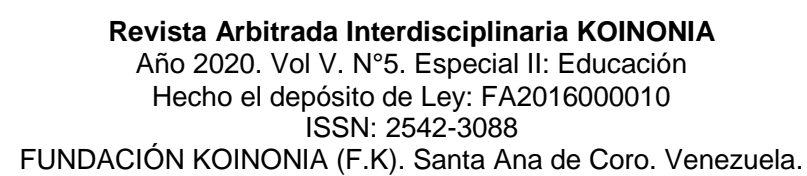

Doris Franco-González; Darwin Gabriel García-Herrera; Claudio Fernando Guevara-Vizcaíno; Juan Carlos Erazo-Álvarez

Se requiere motivación de parte del docente y la predisposición de emigrar el trabajo tradicional, el adaptarse a los cambios y aprender de los estudiantes, una de la propuesta a este cambio es por medio de oficio a la Junta Académica la inserción de la herramienta Scratch en la sección básica como proyecto escolar, y para Primero de Bachillerato con principios a la robótica. La implementación del software como recursos pedagógicos, la herramienta Scratch se vuelve necesaria para el desarrollo de la lógica y la capacidad creativa aumentaría.

Otra de la propuesta de este trabajo investigativo es alfabetizar digitalmente al estudiante, este aspecto es importa, tiene como objetivo enseñar y evaluar los procesos y manejo de la herramienta la motivación la concentración el crear, es parte de la programación que abarca el proceso de aprendizaje en otras áreas.

\section{CONCLUSIONES}

El reto para el docente es mejorar sus prácticas educativas, mediante la aplicación de modelos metodológicos como el $\mathrm{ABP}$, el pensamiento visual y el $\mathrm{AC}$, con esta información da comienzo una serie de proyectos por el avance de nuestra juventud que no se estanquen. serán los guías para la adquisición de conocimientos en todas las fases de la programación, Scratch permite que se pueda aprender a programar como si fuera un juego. Con la utilización de esta herramienta Scratch programar será más divertida permitirá crear, programar y compartir producciones multimedia en un entorno digital online, dando las pautas para su autonomía en la generación de proyectos propios con la creación de su propio software o medios digitales, adquiriendo una participación activa con una mentalidad competitiva.

El desarrollo de esta habilidad se ha tornado difícil en el triángulo educativo por su complejidad, debido a varios factores, uno de ellos son los gobiernos de turno por su pésima asesoramientos en el sistema educativo, no se pega a la realidad social perjudicando a niños y adolescentes principalmente de bajos recursos económicos, otro de las causas es la escasa utilización de herramientas tecnológicas por parte de los 
Doris Franco-González; Darwin Gabriel García-Herrera; Claudio Fernando Guevara-Vizcaíno; Juan Carlos Erazo-Álvarez

docentes, una sociedad digitalizada genera proyectos transformadores de políticas, económicas y sociales.

\section{FINANCIAMIENTO}

No monetario

\section{AGRADECIMIENTO}

Al Colegio Henríquez Coello de la Ciudad de Machala; por apoyar el desarrollo de la investigación.

\section{REFERENCIAS CONSULTADAS}

Aguilar-Yalamá, V. H., \& Pinto-Garzón, G. A. (2015). Competencias digitales y su aplicación en el proceso de enseñanza- aprendizaje de la asignatura de matemática en los estudiantes de décimos años de educación básica superior de la Unidad Educativa Liceo Policial de la ciudad de Quito D.M en el año lectivo 2014 - 2015 [Digital competences and their application in the teaching-learning process of the subject of mathematics in students of tenth years of higher basic education of the Educational Unit Liceo Policial of the city of Quito D.M in the school year 2014 - 2015]. Obtenido de https://cutt.ly/Lhhvvl3

Balanskat, A., \& Engelhardt, K. (2015). A New Model for Coding in Schools (E. Schoolnet, Ed.) Obtenido de https://cutt.ly/ohhQvAA

Brennan, K., \& Resnick, M. (2012). Using artifact-based interviews to study the development of computational thinking in interactive media design. Paper presented at annual American Educational Research Association meeting, Vancouver, BC, Canada. https://cutt.ly/VhhvCVy

Contrera-Espinoza. (2017). Juegos digitales y gamificación aplicados en el ámbito de la educación [Digital games and gamification applied in the field of education]. RIED. Revista Iberoamericana de Educación a Distancia, 19(2), 27-33. https://doi.org/10.5944/ried.19.2.16143 
Doris Franco-González; Darwin Gabriel García-Herrera; Claudio Fernando Guevara-Vizcaíno; Juan Carlos Erazo-Álvarez

Cózar-Gutiérrez, R., González-Calero, S. J., Merino-Armero, J. M., \& Villena-Taranilla, R. (2019). Repositório Científico. Aprendiendo con robots en edades tempranas [Learning with robots at an early age'], 3-45. Brussels. https://doi.org/10.34629/ipl.eselx.cap.livros.026

García-Jiménez, S., \& Roig-Vila, R. (2018). Studio-Based Learning, una metodología al servicio de la enseñanza de los lenguajes de programación [Studio-Based Learning, a methodology at the service of teaching programming languages]. http://hdl.handle.net/10045/70318

Instituto Nacional de Evaluación Educativa, INEVAL. (2019). La educación en Ecuador: logros alcanzados y nuevos desafíos. Resultados educativos 2017-2018 [Education in Ecuador: achievements and new challenges. Educational results 2017-2018]. Recuperado de https://n9.cl/g4rfm

Marín-Gutiérrez, I., Rivera-Rogel, D., \& Ceely-Alvarado, S. (2014). Estudio sobre formación en competencia audiovisual de profesores y estudiantes en el sur de Ecuador [Study on training in audiovisual competence of teachers and students in $\begin{array}{llll}\text { southern } & \text { Ecuador]. } & \text { Cuadernos.info, } & \text { (35), }\end{array}$ 131. https://dx.doi.org/10.7764/cdi.35.628

Miranda-Pinto, M. (2019). Programación y Robótica en Educación Infantil: Estudio Multi Caso en Portugal [Robotic Programming in Children's Education: Multihcast Study in Portugal]. Revista Prisma Social, (25), 248-276.

Monjelat, N., \& San Martín, P. S. (2016). Programar con Scratch en contextos educativos: ¿Asimilar directrices o co-construir Tecnologia. Praxis, 20(1), 61-71. http://dx.doi.org/10.19137/praxiseducativa-2016-200106

Peñaherrera-León, M. (2012). Uso de TIC en escuelas públicas de ecuador: análisis, reflexiones y valoraciones [Use of ICT in public schools in Ecuador: analysis, reflections and evaluations]. Edutec. Revista Electrónica De Tecnología Educativa, (40), a201. https://doi.org/10.21556/edutec.2012.40.364

Pindo, F., Elias, C., Barbot, A., Lopes, J. B., Soares, A., \& Catarino, P. (2016). Using Scratch Software With Students With Special Educational needs in Teaching Natural Sciences And Mathematics. Library, 4058 - 4065. doi: $\underline{10.21125 / \text { inted.2016.1988 }}$ 
Revista Arbitrada Interdisciplinaria KOINONIA

Año 2020. Vol V. N5. Especial II: Educación

Hecho el depósito de Ley: FA2016000010

ISSN: 2542-3088

FUNDACIÓN KOINONIA (F.K). Santa Ana de Coro. Venezuela.

Doris Franco-González; Darwin Gabriel García-Herrera; Claudio Fernando Guevara-Vizcaíno; Juan Carlos Erazo-Álvarez

Quiles-Carrillo, LJ.; Fombuena-Borrás, V.; Balart-Gimeno, RA.; Boronat-Vitoria, T.; Montañés-Muñoz, N. (2019). ¿Estudias o trabajas? - El aprendizaje basado en proyectos como una experiencia enriquecedora de trabajar y estudiar [Do you study or work? - Project-based learning as an enriching experience of working and studying]. En IN-RED 2019. V Congreso de Innovación Educativa y Docencia en Red. Editorial Universitat Politècnica de València. 1052-1063. https://doi.org/10.4995/INRED2019.2019.10472

Terneus-Páez, F., Tobar-Quevedo, J., Loza-Montevelle, D., \& Naranjo-Herera, F. (2 de 5 de 2019). Desarrollo de competencias a través del uso de las herramientas Scratch y Arduino en niños y jóvenes pertenecientes a zonas urbanas marginales del Distrito Metropolitano de Quito. Revista Vínculos, 4(2). http://dx.doi.org/10.24133/vinculosespe.v4i2.155

Vidal, C., Cabezas, C., Parra, J., \& López, L. (2015). Experiencias Prácticas con el Uso del Lenguaje de Programación Scratch para Desarrollar el Pensamiento Algorítmico de Estudiantes en Chile [Practical Experiences with the Use of Scratch Programming Language to Develop the Algorithmic Thinking of Students in Chile]. Formación universitaria, 8(4), 23-32. https://dx.doi.org/10.4067/S0718$\underline{50062015000400004}$

Ziehlke, M. N., \& Talay, C. A. (2017). Programación en entornos visuales. técnicas para la iniciación a la programación y una refeencias realizadas con distintas franjas etarias [9945 Programming in visual environments. techniques for initiation]. Argentina, Argentina. Obtenido de https://cutt.ly/XhhvQaF

C2020 por los autores. Este artículo es de acceso abierto y distribuido según los términos y condiciones de la licencia Creative Commons Atribución-NoComercial-Compartirlgual 4.0 Internacional (CC BY-NC-SA 4.0)

(https://creativecommons.org/licenses/by-nc-sa/4.0/). 